\title{
Advances in diagnostic and therapeutic endoscopy
}

\author{
Mohamed Hussein \\ Laurence Lovat \\ Rehan Haidry
}

Mohamed Hussein BSc(Hons) MRCP is a Specialist Registrar in Gastroenterology at University College London Hospital and a Research Fellow in the Division of Surgery and Interventional Sciences, UCL, London, UK. Competing interests: None

Laurence Lovat BSc PhD FRCP is a Professor of Gastroenterology and Biophotonics, Division of Surgery and Interventional Sciences, UCL, London, UK. Competing interests: None

Rehan Haidry BSc (Hons) MD FRCP is a Consultant in Gastroenterology, Clinical Lead for GI Medicine and Director of Endoscopy at University College Hospital, London, UK. Competing interests: None. RJH has received Educational grants to support research from Medtronic Ltd, Cook Endoscopy (fellowship support), Pentax Europe, C2 Therapeutics, Beamline diagnostics.

\begin{abstract}
The requirement for efficient endoscopic visualization and instrumentation has increased over the past decade given the considerable burden that luminal disorders of the gastrointestinal tract exert on individuals and healthcare economies. The push for greater diagnostic yield has driven advances in optical physics and bioengineering, which are revolutionizing diagnostic and therapeutic endoscopy. This article highlights emerging technologies since our last review, focusing on advances in imaging and endoscope design, and how these are shaping the therapeutic approach to diseases of the human gastrointestinal tract. Their application to improving diagnostic ability and patient care, as well as their limitations, are discussed.
\end{abstract}

\section{Keywords}

Ablation; colonoscopy; endoscopic resection; endoscopy; therapeutic endoscopy; third space endoscopy

\section{Key points}

- Virtual chromoendoscopy includes narrow-band imaging, i-Scan ${ }^{\mathrm{TM}}$, blue laser imaging and linked colour imaging. These modalities can enhance the detection of previously invisible lesions so they are now amenable to endoscopic treatment

- Computer-aided detection and diagnosis, using deep learning methodology, is currently under development and has the potential to revolutionize endoscopy

- The development of imaging has complemented the advances in therapeutic endoscopy. Improved lesion recognition allows the use of advanced therapies such as radiofrequency ablation, cryotherapy, endoscopic mucosal resection and submucosal dissection to potentially cure early cancers, thereby improving the quality of patient care

- Third space endoscopy has developed over the last decade, creating a new operating field to treat gastrointestinal conditions such as Zenker's diverticulum, achalasia and gastroparesis.

\section{Introduction}

High-definition (HD) digital technology has moved endoscopic imaging to a new dimension. Endoscopic innovations have arisen from the explosion of technical achievements through the interaction between physicians and engineers, and the incorporation of technology from other fields such as computing, artificial intelligence and physics. These endoscopic advances are important to meet the need that we are still missing upper gastrointestinal (GI) cancers and colonic polyps. A meta-analysis showed that $11.3 \%$ of upper GI cancers were missed during endoscopy up to 3 years before diagnosis. ${ }^{1}$ 
Hand in hand with these advancements in imaging, there is a continuing evolution of the ergonometric design of endoscopes, with dedicated accessories that allow increasing applications of therapeutic endoscopy. The endoscopic treatment of early cancers of the GI tract continues to advance at a pace and is being constantly driven by improved imaging.

\section{Advances in imaging \\ Improved digital imaging and virtual chromoendoscopy}

Video endoscopes use white light from a xenon or halogen source for illumination and rely on a chargecoupled device (CCD) chip to enhance image resolution and magnification, in order to reconstruct the images. Standard definition white light endoscopy (WLE) has been rapidly replaced by the introduction of HD endoscopes. Whereas CCD chips produce an image signal of 100,000-400,000 pixels that is displayed in SD format, the chips currently in use in HD endoscopes produce resolutions that range from 850,000 to 1.3 million pixels. These latest innovations have exponentially increased the endoscopist's ability to inspect and visualize subtle mucosal details.

Manipulation of the image using additional optical technologies, such as i-Scan ${ }^{\mathrm{TM}}$ (Pentax, Japan), narrow-band imaging (NBI; Olympus, Japan) and blue laser imaging (BLI; Fujinon, Japan), can further enhance the detection of previously invisible small lesions and is often referred to as virtual chromoendoscopy (Table 1).

Narrow-band imaging: NBI is based on the theory that the depth of penetration of light depends on its wavelength. Blue light penetrates superficially, whereas red light penetrates more deeply. Olympus Medical Systems uses optical filters to isolate blue $(415 \mathrm{~nm})$ and green $(540 \mathrm{~nm})$ light. These two bands of light are absorbed by superficial mucosal structures and better absorbed by haemoglobin, providing enhanced visualization of superficial mucosal structures and blood vessels. A recent study showed that an extra 30 seconds' observation with bright high-resolution NBI decreased the rate of missed polyps in the right side of the colon (see Further reading).

i-Scan: the i-Scan ${ }^{\mathrm{TM}}$ optical enhancement (OE) function uses processor technology that combines digital signalling in a similar way to $\mathrm{i}-\mathrm{S} \mathrm{can}^{\mathrm{TM}}$, and optical filters that limit the special characteristics of the illumination lights. The optical filters achieve higher overall transmittance by connecting the peaks of the haemoglobin absorption spectrum $(415 \mathrm{~nm}, 540 \mathrm{~nm}, 570 \mathrm{~nm})$, which displays the surface structures of blood vessels, glandular ducts and mucosal membranes in higher contrast than WLE. This technology allows for improved characterization, detection and diagnosis of Gl tract lesions (Figure 1). i-Scan ${ }^{\text {TM }}$ OE has been shown to improve detection of Barrett's oesophagus dysplasia on endoscopic imaging and the accuracy of histology prediction compared with HD WLE. ${ }^{2}$

Blue laser imaging: $\mathrm{BLI}$ is a recently developed system that uses a combination of two types of monochromatic laser light, a $450 \mathrm{~nm}$ laser providing white light and a $410 \mathrm{~nm}$ laser which allows visualization of vascular microarchitecture similar to NBI. It combines white light with NBI, producing a bright high-resolution image of the mucosa (Figure 2). It has been shown that this BLI bright technique has a higher real-time detection rate for early gastric cancer than WLE. ${ }^{3}$

Linked colour imaging (LCl): $\mathrm{LCl}$ (Fujinon) is a new post-processing technology that enhances colour contrast. It integrates short wavelength narrow-band laser light with white laser light based on BLI bright mode. Using LCl, white appears whiter, and red appears redder, making it easier to detect colour changes in the mucosa (Figure 3). A randomized controlled trial showed that $\mathrm{LCl}$ was superior to conventional WLE for the detection of colonic sessile serrated adenomas/polyps (see Further reading).

The recently developed ELUXEO ${ }^{\mathrm{TM}}$ video processor VP-7000 (Fujinon), powered by four- LED multilight technology, allows endoscopists to switch between WLE, BLI and LCI modes. 


\section{Real-time in vivo diagnosis}

Even with advancements in endoscopic imaging, current practice still relies widely on endoscopic sampling of suspicious areas for histological confirmation of neoplasia. New technologies now exist that allow in vivo diagnosis of cellular atypia and guide therapy.

Confocal laser endomicroscopy: this is a developing technology that enables the high-resolution in vivo imaging of tissue microstructures at or near the level of histopathology without requiring tissue excision. It is based on the principle of illuminating tissue with a low-power laser and then detecting the fluorescent light reflected from the tissue. Magnification of $>1000$-fold can be achieved to view cellular structures. Images are interpreted based on the cellular architecture and capillary structures. Tissue fluorescence is achieved using intravenously or topically applied contrast agents, usually intravenous fluorescein.

Two devices are currently available for clinical and research purposes: an endoscope-based system that is fully integrated into the tip of a conventional endoscope (OptiScan, Australia; Pentax) but only currently available for research purposes; and a probe-based system that can be passed down the working channels of a range of standard endoscopes (Cellvizio®; Mauna Kea Technologies, France), which is available commercially.

Spectroscopy and 'optical biopsies': spectroscopy is based on light interaction with tissue. The incident light directed on the tissue can be reflected in different patterns, called 'scattering events'. Light can be also be absorbed and re-emitted at longer wavelengths, or shifted slightly to a different wavelength. These characterizations can correlate with histopathology, subcellular architecture (e.g. enlarged nuclei), specific molecular bonds or differing tissue absorptions of light driven by exogenous or endogenous fluorescent compounds (fluorophores; see below). Fourier transform infrared spectroscopy can detect specific molecules from their unique vibrational absorption spectra in human tissue. It has been shown that it can accurately differentiate between columnar mucosa Barrett's oesophagus and normal squamous oesophagus.

Autofluorescence: the use of autofluorescence during endoscopy is based on the principle that the mucosa contains variable amounts of fluorophores (biological substances that emit fluorescent light when exposed to light of a shorter wavelength). Different tissues have varied fluorescence emission levels, and therefore normal mucosa appears green under fluorescence excitation, whereas dysplastic or neoplastic tissue appears purple. Autofluorescence has been found to be useful in combination with different types of imaging modalities, but not useful as a stand-alone primary imaging technique because of its low sensitivity and high false-positive rate.

\section{Molecular imaging}

This emerging optical imaging method allows tissue to be assessed in a non-destructive way using receptor expression within the mucosa on a cellular level. It uses fluorescently labelled probes with high specificity towards particular molecular targets. This allows localization of dysplastic lesions/tumours, visualization of the molecular characteristics and activity of molecules, and the biological process that influences tumour behaviour and its response to therapy.

\section{Optical coherence tomography (OCT)}

OCT is a novel technique that relies on light back-scattering to obtain both cross-sectional and threedimensional images of tissue microstructures. These images are visually analogous to viewing a coarse black and white histological specimen. OCT uses reflected light to construct an image, similar to the use of acoustic waves in ultrasound. To date, Gl tract scanning has been achieved by inserting a probe through the working channel of a regular endoscope. Although neither a water interface nor tissue apposition is required, the depth of scanning achieved is limited to $1-2 \mathrm{~mm}$ because of light scattering by the tissues.

\section{Computer-assisted imaging analysis}

Computer-aided detection and diagnosis combine a computer's ability to learn and perform endoscopic tasks using advances in machine learning, and deep learning methodology. A recent study showed that a convolutional neural network can be used to classify oesophageal squamous dysplasia into normal or 
abnormal. This system can be used as an in vivo, real-time clinical decision-making support tool for endoscopists assessing squamous dysplasia and deciding on therapy (see Further reading). This technology has the potential to revolutionize endoscopy.

\section{Advances in endoscope design}

Improved colonoscopic field of vision

G-EYE colonoscope: The G-EYE ${ }^{\mathrm{TM}}$ (Pentax) consists of a reusable balloon attached to the distal end of the colonoscope, which flattens the colonic folds, centralizes the optical images and reduces bowel slippage. There is enhanced visualization of the colon with the insufflated balloon, thus increasing the adenoma detection rate. Once a polyp has been detected, balloon anchoring in the colon facilitates fast and accurate polyp removal. A recent randomized controlled study showed that G-EYE ${ }^{\mathrm{TM}}$ colonoscopy is superior to standard colonoscopy for increasing the rate of adenoma detection. ${ }^{4}$

Full-spectrum view colonoscopy: Full Spectrum Endoscopy® (Fuse ${ }^{\circledR}$; EndoChoice, USA) consists of a standard colonoscope with two lateral lenses and one on the forward tip. This increases the maximum field of vision up to $330^{\circ}$, compared with $<170^{\circ}$ on a standard forward-viewing colonoscope. Fuse ${ }^{\circledR}$ has been shown to increase adenoma detection rate.

EndoCuff ${ }^{\mathrm{TM}}$ (Arc Medical Design, Leeds, UK): this is a single-use device that attaches to the distal tip of the colonoscope (Figure 4). It is a soft plastic cap involving a cylindrical core with flexible finger-like projections made of thermoplastic elastomer. On withdrawal, the traction on the mucosa allows the projections to extend radially, therefore manipulating the colon and allowing careful inspection of the proximal aspect of the folds. It has been shown to significantly improve adenoma detection rate in bowel cancer screening patients.

EndoRing ${ }^{\mathrm{TM}}$ (EndoAid Ltd, Caesarea, Israel): this comprises a single-use silicon rubber device attached to the distal end of the colonoscope (Figure 4). It consists of flexible circular rings that stretch the colonic folds during withdrawal. In a randomized trial comparing HD forward-viewing colonoscopy with the Fuse ${ }^{\circledR}$, EndoCuff ${ }^{\mathrm{TM}}$ and EndoRing ${ }^{\mathrm{TM}}$ devices, the EndoCuff ${ }^{\mathrm{TM}}$ was superior to the EndoRing ${ }^{\mathrm{TM}}$, and HD forwardviewing colonoscopy was superior to the Fuse® system. ${ }^{5}$

\section{Digital single-operator cholangioscopy}

SpyGlass $^{\mathrm{TM}}$ DS Direct Visualization System (digital SOC, DS System; Boston Scientific, USA) was introduced in 2015. This catheter-based system attaches to the endoscope during endoscopic retrograde cholangio-pancreatography and provides a direct intraluminal view of the biliary ducts, offering advanced possibilities for diagnosis and intervention (Figure 5). This provides several advantages over the conventional fibre-optic SpyGlass ${ }^{\mathrm{TM}}$ system, including newly added suction and injection functions, a tapered tip that allows easier insertion into the biliary system, a $120^{\circ}$ digital field of view and up to 4 -fold higher resolution.

\section{Double balloon enteroscopy}

Developed in Japan in 2001, this is the first device to allow visualization of the whole Gl tract in real time. It allows for visualization and the application of therapeutics in the small bowel, therefore avoiding open surgery. It consists of a high-resolution endoscope with an overtube. Balloons are attached to the tip of the endoscope and to the overtube. Using various manoeuvres, the small bowel is sequentially pulled onto the overtube in small segments. This procedure can be performed via either the oral or the anal route.

\section{Magnet-assisted capsule endoscopy}

Capsule endoscopy was introduced in 2000 and provided a more comfortable diagnostic alternative to standard endoscopy. However, there was difficulty in assessing the stomach because of the large size of the gastric cavity. To address this, magnet-controlled capsule endoscopy has been developed in China; this uses a magnetic field to control and steer the direction of the capsule, a magnet inside the capsule initiating 
the movement. It has been found to detect focal lesions in the stomach with comparable accuracy to standard endoscopy (see Further reading). It has also shown positive results in the colon.

\section{Advances in therapeutic endoscopy}

The advances in imaging and diagnostic techniques have paved the road for the application of new interventional modalities to manage the new pathologies uncovered by the above advances. This can now be done with minimally invasive techniques, therefore reducing mortality and morbidity. The lymphovascular distribution of the human GI tract informs us that pathology and neoplasia confined to the superficial layers, such as the mucosa and submucosa, can be successfully treated with endoscopic therapy because the long-term risk of nodal metastases is minimal.

\section{Radiofrequency ablation (RFA)}

The Barrx ${ }^{\mathrm{TM}}$ radiofrequency ablation system(Covidien) is used for the treatment of superficial neoplasia in the oesophagus. Balloon-based or focal endoscope-mounted devices are used to provide a pulse of radiofrequency energy to ablate the mucosal surface of the oesophagus. Both Barrett's oesophagus with related neoplasia and early squamous cell neoplasia have been treated successfully and safely using this intervention. The use of RFA in treating mucosal haemorrhagic conditions such as radiation proctopathy and gastric antral vascular ectasia is, however, less well established.

\section{Cryoablation}

Cryoablation involves the use of extreme cold to destroy diseased tissue. Liquid nitrogen at $-176^{\circ} \mathrm{C}$ is delivered to the mucosa, causing denaturing of proteins and fracturing of cell membranes. Within a few days, the tissue sloughs off, heals and is replaced with non-squamous epithelium (Figure 6). It has been shown that patients undergoing treatment with cryotherapy are equally as likely as those undergo RFA to achieve complete eradication of dysplasia.

\section{Third space endoscopy}

The submucosal space is an operating field used for the treatment of variety of conditions including achalasia, gastroparesis, Zenker's diverticulum and submucosal tumours:

\section{Endoscopic submucosal dissection}

This technique was developed in the early 20th century in Japan, initially as a curative treatment for gastric cancer, but now also as an effective treatment for oesophageal and colon cancers. It allows the removal of submucosal tumours arising from the muscularis propria by using the submucosal tunnelling technique. En bloc excision of tumours using this technique allows tumours to be completely staged and also provides a cure for early cancer. The problems associated with it are the difficulty of training physicians, long procedure time and rate of complications.

E-POEM: (O)Esophageal per-oral endoscopic myotomy (E-POEM) is a novel modality for the treatment of achalasia that is performed by gastroenterologists and surgeons. It represents a natural orifice transluminal endoscopic surgery approach to Heller myotomy. E-POEM has the minimal invasiveness of an endoscopic procedure that can duplicate results of the surgical Heller myotomy. The technique of E-POEM is centred on creation of a submucosal tunnel within the distal oesophagus, in which a myotomy is performed with dissection of the inner circular muscle of the oesophagus and minimal dissection of the circular muscle of the lower oesophageal sphincter. E-POEM has been shown to be safe, effective and durable for the early treatment of achalasia. A randomised control trial comparing E-POEM versus pneumatic dilatation showed $\mathrm{POEM}$ is associated with a higher one year therapeutic success rate.

G-POEM: pylorospasm has been associated with the development of gastroparesis, so pylorus- based treatments have been recommended for the treatment of this patient cohort. Gastric per-oral endoscopic myotomy (G-POEM) of the pylorus is a novel technique proposed for the treatment of refractory gastroparesis in selected patients. It involves submucosal injection along the greater curve of the stomach proximal to the pylorus, mucosal incision, submucosal tunnelling, myotomy involving the pyloric muscle and then closure of the incision using endoclips. There is high quality evidence for this procedure and recent metanalysis have shown high clinical success rate and technical success rates of $100 \%$. 
Submucosal tunnelling endoscopic septum division (Z-POEM): this is for the treatment of Zenker's diverticulum, a pouch that can form at the junction of the hypopharynx and oesophagus and cause symptoms of dysphagia. It involves endoscopic division of the septum between the diverticular and oesophageal lumen. This avoids the higher risks of open surgery.

\section{Bariatric endoscopy}

Endoscopic sleeve gastroplasty: this is a new weight loss procedure involving a full -thickness suture to invaginate the greater curvature of the stomach, extending from the incisura to the gastro-oesophageal junction. This creates a narrow luminal sleeve with a small fundic pouch, and decreases the gastric volume by up to $70 \%$. Endoscopic sleeve gastroplasty has been shown to be safe, well tolerated and effective. It has been reported to provide sustained weight loss for up to 24 months, reducing markers of hypertension, diabetes mellitus and hypertriglyceridaemia (see Further reading).

Aspirational therapy: in this, an AspireAssist ${ }^{\circledR}$ system (Aspire Bariatrics, USA) is connected to an A-tube inserted into the stomach in a similar fashion to a percutaneous endoscopic gastrostomy tube. This facilitates the removal of a portion of gastric contents 20-30 minutes after each meal for weight loss. It removes approximately $30 \%$ of the calories consumed during a meal.

EndoBarrier ${ }^{\circledR}$ : the EndoBarrier® (GI Dynamics, USA) duodenal-jejunal bypass liner is endoscopically anchored in the duodenum under fluoroscopy. The proximal part of the small bowel is covered with an impermeable fluoropolymer sleeve open at both ends up to $60 \mathrm{~cm}$ into the jejunum. This creates a mechanical barrier allowing food to bypass the duodenum and $60 \mathrm{~cm}$ of the jejunum, so it does not mix with pancreaticobiliary secretions until later in the GI tract.

Gastro-duodeno-jejunal bypass sleeve (GJBS): the GJBS (ValenTx, Inc. Carpinteria, CA, USA) is a 120 $\mathrm{cm}$ fluoropolymer sleeve anchored to the gastro-oesophageal junction that extends from the stomach to the jejunum. This allows food to bypass the stomach, duodenum and proximal jejunum.

Duodenal mucosal resurfacing: this is a new endoscopic procedure as part of an ongoing trial to treat poorly controlled type 2 diabetes. It involves thermal ablation of the duodenum and a remodelling of cells involved in hormone production related to sugar control. Under endoscopic guidance, a guidewire is inserted into the distal duodenum. A catheter is delivered over a wire. Under endoscopic visualization, a balloon is expanded and filled with hot fluid to allow circumferential thermal ablation. (Figure 7). This procedure has been associated with a fall in glycated haemoglobin concentrations. 


\begin{tabular}{|c|c|c|c|}
\hline \multicolumn{4}{|c|}{ Digital imaging techniques } \\
\hline Modality & Mechanism & Advantages & Limitations \\
\hline $\begin{array}{l}\text { Narrow- } \\
\text { band } \\
\text { imaging }\end{array}$ & $\begin{array}{l}\text { Taking into account absorptive } \\
\text { and reflective properties of the } \\
\text { mucosal surface, optic filters } \\
\text { isolate two specific bands of light } \\
(415 \mathrm{~nm} \text { blue, } 540 \mathrm{~nm} \text { green) to } \\
\text { enhance visualization }\end{array}$ & \multirow{4}{*}{$\begin{array}{l}\text { These techniques allow for } \\
\text { significantly improved } \\
\text { mucosal visualization by } \\
\text { highlighting subtle } \\
\text { architectural or vascular } \\
\text { differences on the mucosal } \\
\text { surface. This improves } \\
\text { detection of subtle } \\
\text { irregularities and improves } \\
\text { therapeutic outcomes }\end{array}$} & \multirow{4}{*}{$\begin{array}{l}\text { The principal drawback for } \\
\text { all these techniques lies in } \\
\text { the need for careful } \\
\text { observation of the mucosa } \\
\text { followed by detailed } \\
\text { inspection using image } \\
\text { manipulation. This is time- } \\
\text { consuming and requires } \\
\text { considerable expertise }\end{array}$} \\
\hline $\mathrm{i}^{-S} \operatorname{san}^{\mathrm{TM}}$ & $\begin{array}{l}\text { Digital image enhancement } \\
\text { settings such as tonal, contrast } \\
\text { and surface filters improve } \\
\text { visualization of vascularity and } \\
\text { topography }\end{array}$ & & \\
\hline $\begin{array}{l}\text { Blue } \\
\text { laser } \\
\text { imaging }\end{array}$ & $\begin{array}{l}\text { This new system for image- } \\
\text { enhanced endoscopy uses a } \\
\text { combination of two } \\
\text { monochromatic laser lights to } \\
\text { produce a new type of bright, } \\
\text { high-resolution image of mucosa. } \\
\text { A } 410 \mathrm{~nm} \text { laser visualizes } \\
\text { vascular microarchitecture, and a } \\
450 \mathrm{~nm} \text { laser provides white light } \\
\text { by excitation }\end{array}$ & & \\
\hline $\begin{array}{l}\text { Linked } \\
\text { colour } \\
\text { imaging }\end{array}$ & $\begin{array}{l}\text { Post-processing technique } \\
\text { enhances colour contrast }\end{array}$ & & \\
\hline
\end{tabular}

Table 1 


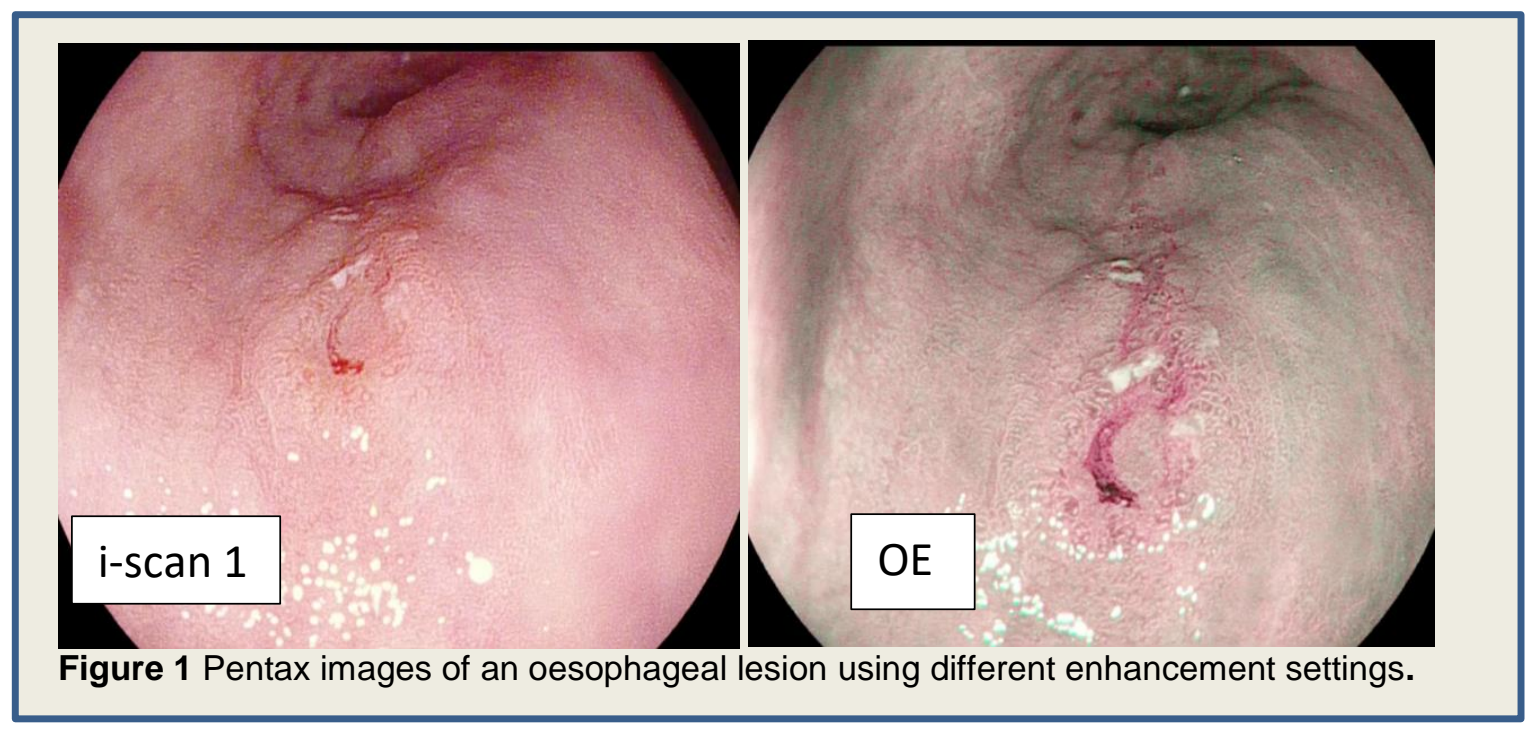




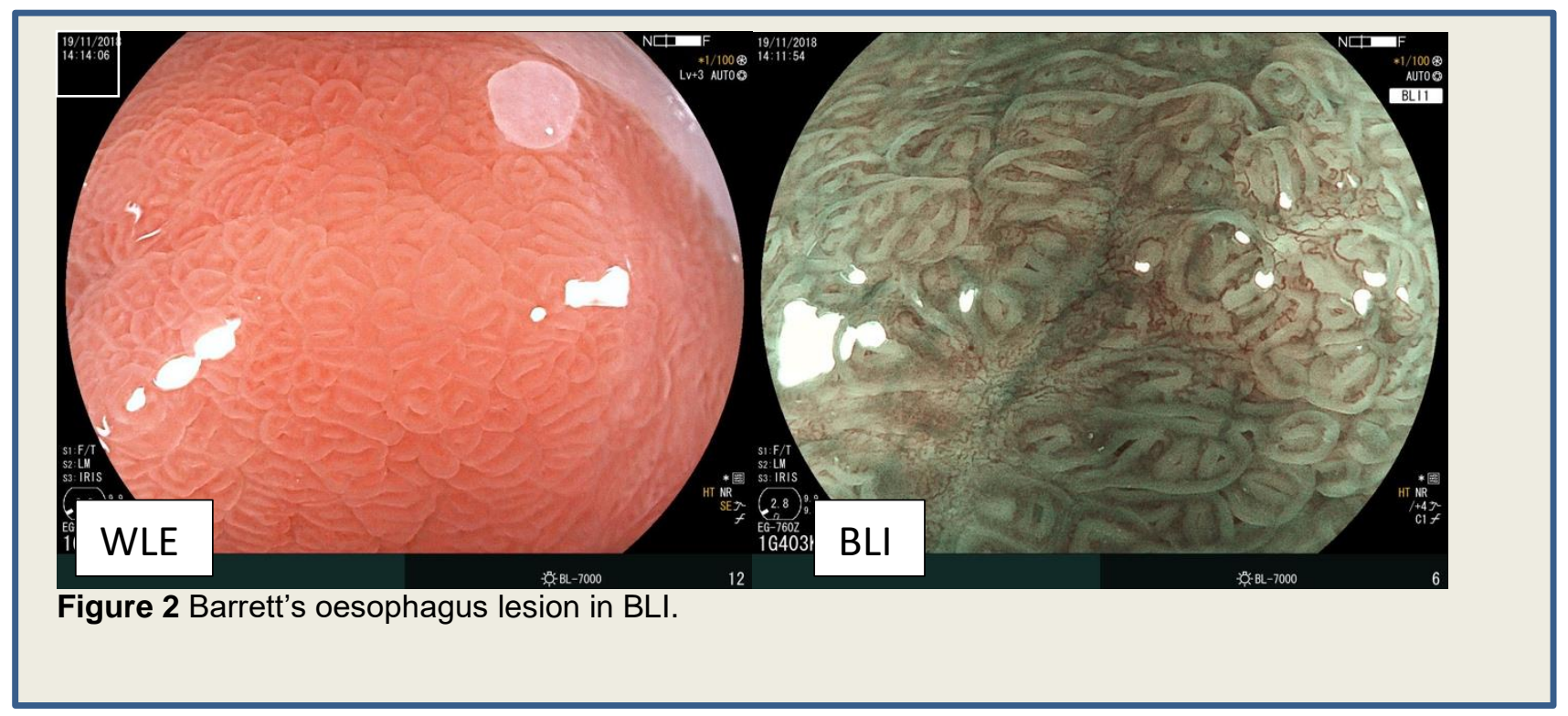




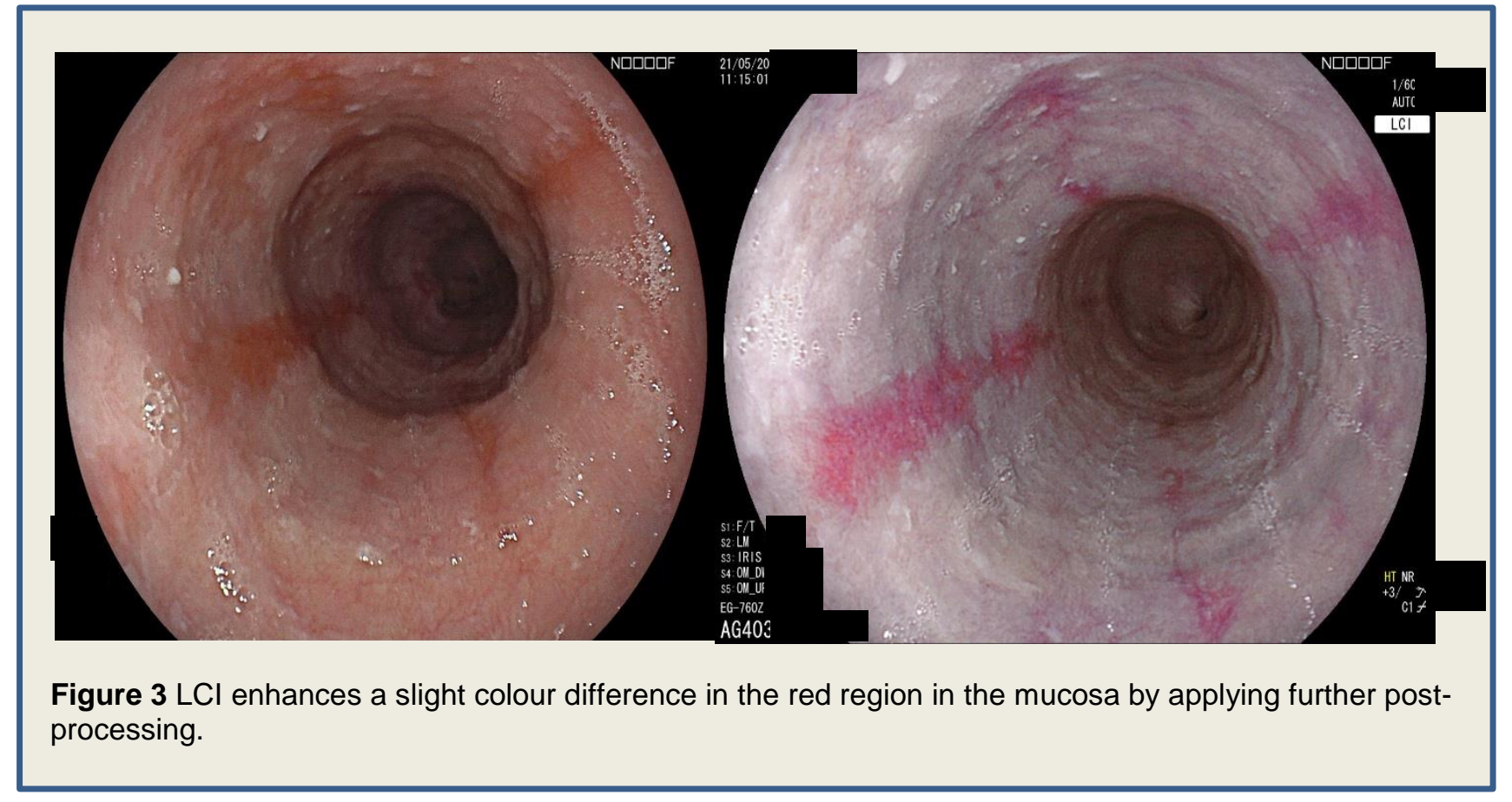




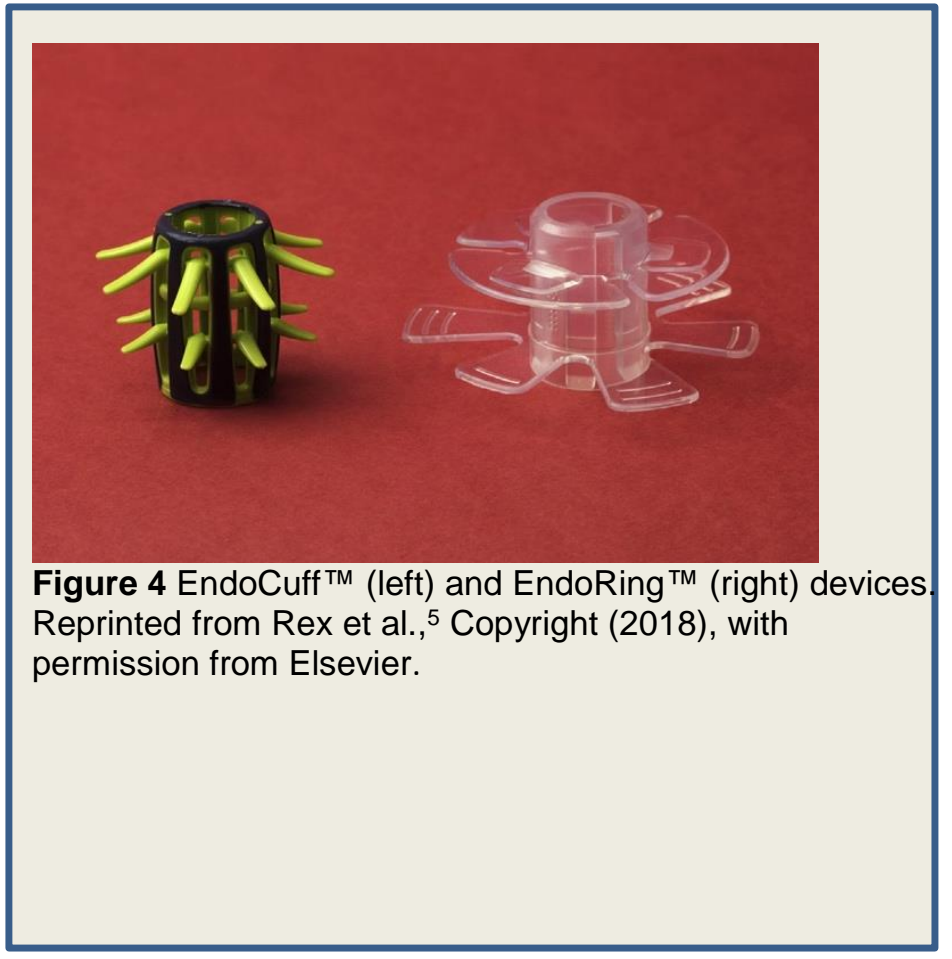




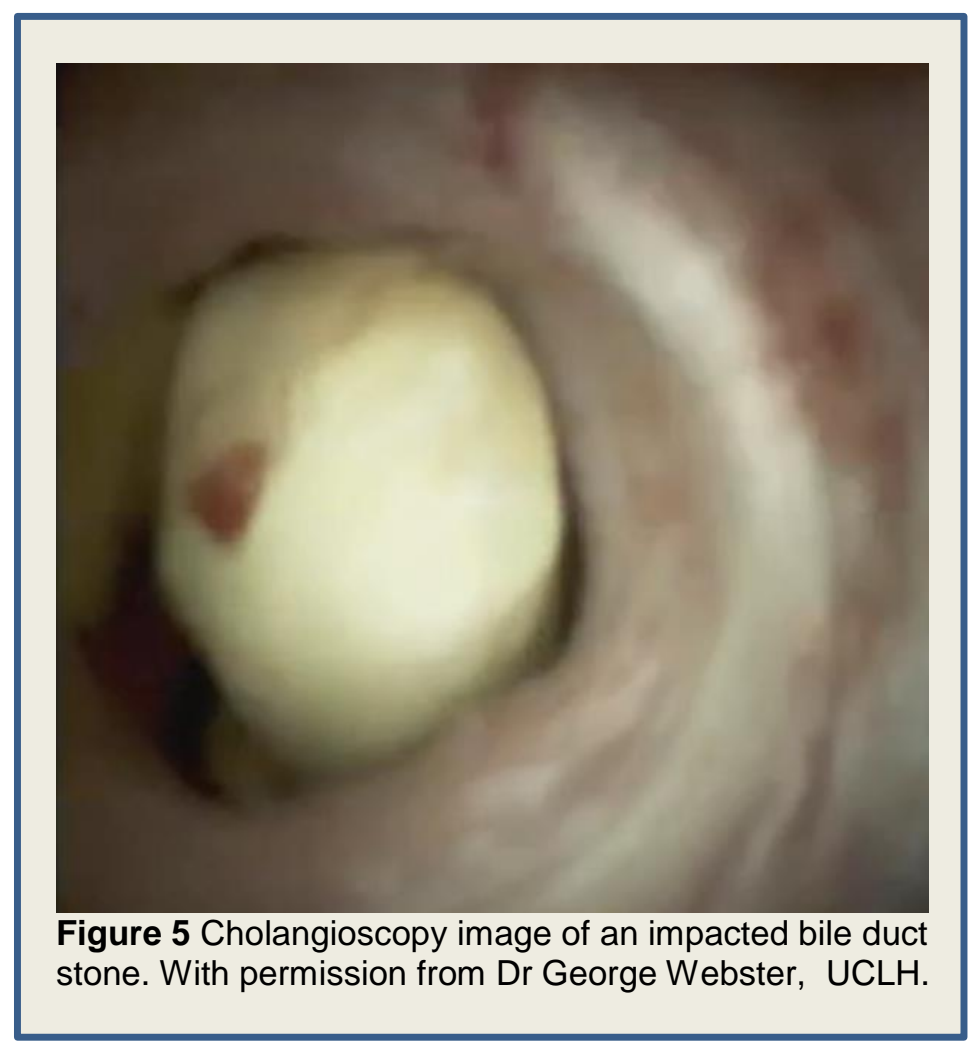




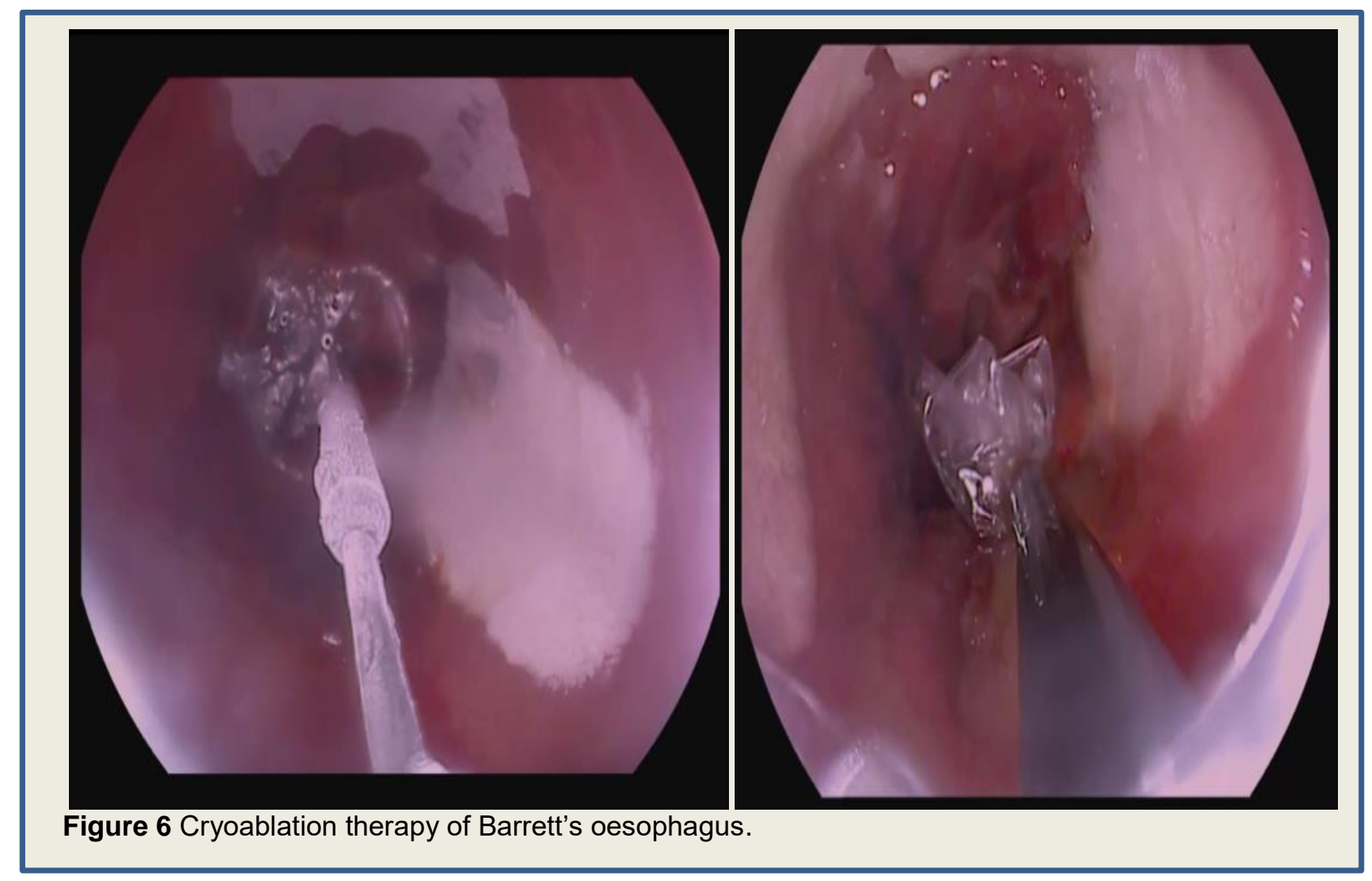




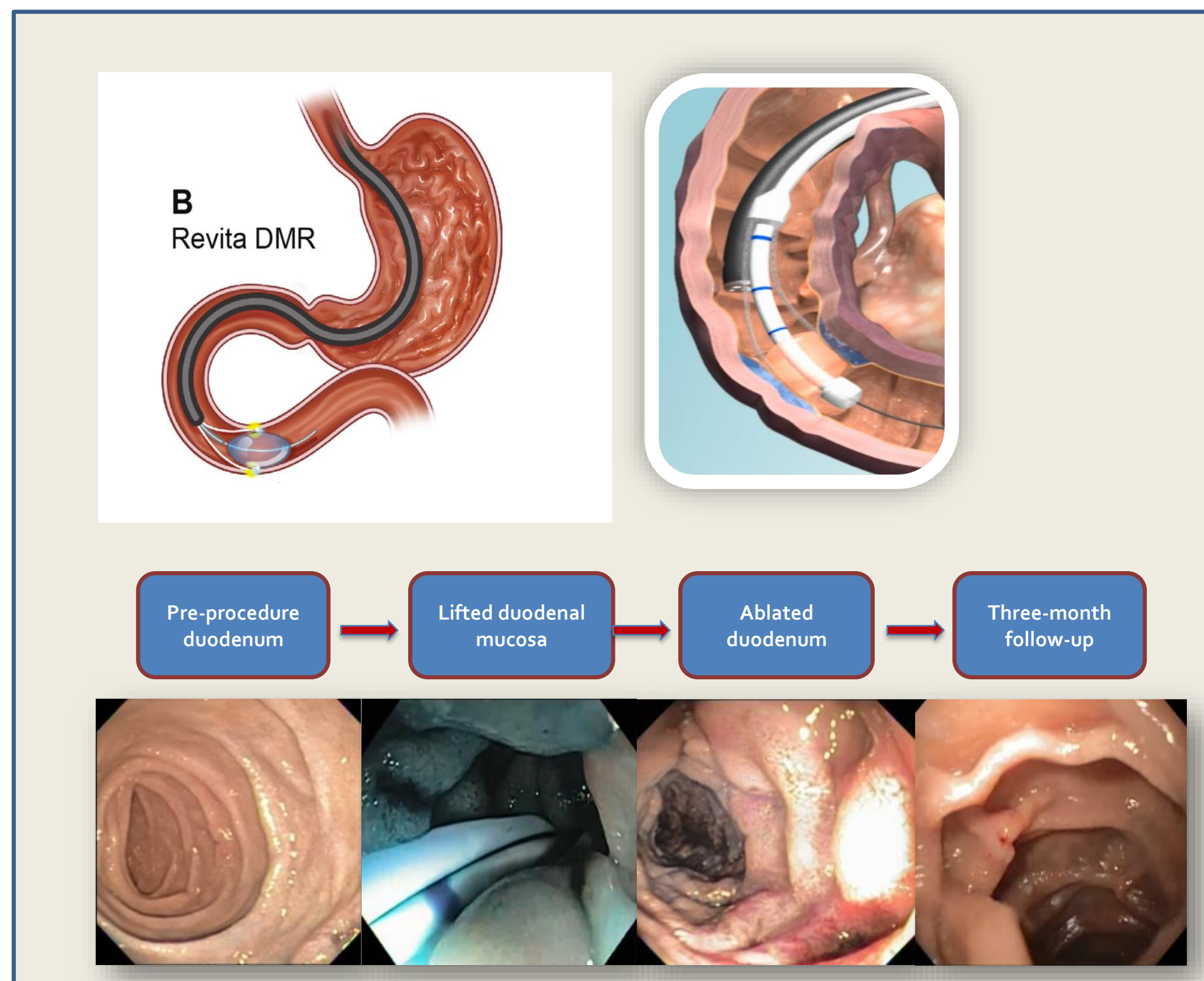

Figure 7 DMR procedure to treat poorly controlled type 2 diabetes via thermal ablation of the duodenal surface. 


\section{KEY REFERENCES}

${ }^{1}$ Menon S, Trudgill N. How commonly is upper gastrointestinal cancer missed at endoscopy? A metanalysis. Endosc Int Open 2014; 2: E46-50.

2 Everson MA, Lovat LB, Graham DG, et al. Virtual chromoendoscopy using optical enhancement improves the detection of Barrett's esophagus associated neoplasia. Gastrointest Endosc 2019; 89 (2): 247-56.

${ }^{3}$ Osamu D, Nobuaki Y, Yuji N, et al. Blue laser imaging-bright improves the real-time detection rate of early gastric cancer: a randomized controlled study. Gastrointest Endosc 2019; 89 (1): 47-57.

${ }^{4}$ Shirin H, Shpak B, Epshtein J, et al. G-EYE colonoscopy is superior to standard colonoscopy for increasing adenoma detection rate: an international randomized controlled trial (with videos). Gastrointest Endosc 2019; 89(3): 545-53.

${ }^{5}$ Rex D, Repici A, Gross S, et al. High-definition colonoscopy versus endocuff versus endorings versus full-spectrum endoscopy for adenoma detection at colonoscopy: a multicenter randomized trial. Gastrointest Endosc 2018; 88(2): 335-344.

\section{FURTHER READING}

Everson M, Herrera LCGP, Li W et al. Artificial intelligence for the real-time classification of intrapapillary capillary loop patterns in the endoscopic diagnosis of early oesophageal squamous cell carcinoma: a proof-of-concept study. UEG Journal 2019; 7(2): 297-306.

Fujimoto D, Muguruma N, Okamoto K, et al. Linked color imaging enhances endoscopic detection of sessile serrated adenoma/polyps. Endosc Int Open 2018; 6(3): E322-34.

Liao Z, Hou X, Lin-Hu EQ, et al. Accuracy of magnetically controlled capsule endoscopy, compared with conventional gastroscopy, in detection of gastric diseases. Clin Gastroenterol Hepatol 2016; 14(9): 1266-73. Sharaiha RZ, Kumta NA, Saumoy M, et al. Endoscopic sleeve gastroplasty significantly reduces body mass index and metabolic complications in obese patients. Clin Gastroenterol Hepatol 2017; 15(4): 504-10.

Yoshida N, Inoue K, Yasuda R, et al. An additional 30-s observation of the right sided colon with narrow band imaging decreases missed polyps: a pilot study. Dig Dis Sci 2018; 63(12): 3457-64

\section{TEST YOURSELF}

To test your knowledge based on the article you have just read, please complete the questions below. The answers can be found at the end of the issue or online here.

\section{Question 1}

A 65-year-old man presented with symptoms of reflux. An endoscopy was performed.

\section{Investigation}

-Endoscopy showed a $10 \mathrm{~cm}$ segment of Barrett's oesophagus, with a nodule at $35 \mathrm{~cm}$. Biopsies showed columnar mucosa with intestinal metaplasia and focal high-grade dysplasia at $35 \mathrm{~cm}$

\section{What is the next most appropriate management step?}
A. Cryoablation
B. Radiofrequency ablation 

C. Endoscopic mucosal resection
D. Repeat endoscopy with surveillance biopsies in 6 months
E. No further endoscopic procedure required and discharge patient.

Correct answer: C. The British Society of Gastroenterology guidelines recommend that endoscopic resection should be the therapy of choice in visible lesions within Barrett's oesophagus. This is also the most accurate staging system for early Barrett's neoplasia. If this was flat Barrett's with no visible lesions, then answers A. or B. would be appropriate. D. is correct in the case of low grade dysplasia or indefinite. For dysplasia. All patients with high grade dysplasia need an MDT discussion and therapeutic intervention as per guidelines. E. is of course an incorrect answer.

\section{Question 2}

A 35-year-old man presented with a 1-year history of dysphagia to solids and liquids, chest pain with eating and regurgitation.

\section{Investigation}

- Oesophageal manometry confirmed achalasia

\section{What is the best endoscopic treatment option that can be offered:}
A. Cryoablation
B. Per Oral Endoscopic Myotomy for Zenkers Diverticulum(Z-POEM)
C. Endoscopic sleeve gastroplasty
D. Oesophageal per-oral endoscopic myotomy (E-POEM)
E. Duodenal mucosal resurfacing

Correct answer: D. E-POEM is a minimally invasive endoscopic procedure for achalasia that can mimic the results of a surgical Heller's myotomy. Z-POEM is an endoscopic treatment for Zenkers diverticulum. Doudenal mucosal resurfacing involves thermal ablation of the duodenum as part of an ongoing trial for management of poorly controlled type 2 diabetes. Endoscopic sleeve gastroplasty is a weight loss procedure which involves full thickness suturing reducing gastric volume by $70 \%$. Cryoablation has no role in the management of achalasia

\section{Question 3}

A 35-year-old woman presented with right upper quadrant pain and liver function test results indicating obstruction. Magnetic resonance cholangiopancreatography (MRCP) showed a dilated common bile duct with large stones. The next day an endoscopic retrograde cholangiopancreatography (ERCP) was performed with sphincterotomy and balloon trawl, but some of the large stones still remained. A stent was inserted, and the patient returned to the ward. An interval MRCP again showed duct dilatation with stones.

\section{What is the best next step:}
A. Standard ERCP
B. Monitor liver function tests
C. Cholangioscopy
D. Refer for surgery
E. Repeat MRCP in 4 weeks

Correct answer: C. Cholangioscopy is used for treating difficult bile stones and assessing indeterminate biliary strictures. Electrohydraulic or laser lithotripsy probes fragment the stones. A standard ERCP procedure has already been tried and had failed. ERCP is the first line procedure in the UK for management of biliary strictures and stones. Answer $\mathbf{B}$. and $\mathbf{E}$. is incorrect as therapeutic intervention is now required in view of confirmed ductal stones. Answer $\mathbf{D}$ is incorrect. NICE guidance suggests that patients with bile duct stones should have an ERCP before a laparoscopic cholecystectomy. 
- 